Journal of Universal Mathematics

Vol.4 No.2 PP.271-282 (2021)

ISSN-2618-5660

DOI: $10.33773 /$ jum.957741

\title{
NONEXISTENCE OF GLOBAL SOLUTIONS FOR A KIRCHHOFF-TYPE VISCOELASTIC EQUATION WITH DISTRIBUTED DELAY
}

\author{
HAZAL YÜKSEKKAYA AND ERHAN PIŞKIN
}

0000-0002-1863-2909 and 0000-0001-6587-4479

\begin{abstract}
In this paper, we consider a Kirchhoff-type viscoelastic equation with distributed delay and source terms. We obtain the nonexistence of global solutions under suitable conditions.
\end{abstract}

\section{INTRODUCTION}

In this paper, we consider the following Kirchhoff-type viscoelastic equation with distributed delay and source terms

$$
\begin{cases}u_{t t}-M\left(\|\nabla u\|^{2}\right) \Delta u+\int_{0}^{t} g(t-s) \Delta u(s) d s & \\ +\mu_{1} u_{t}+\int_{\tau_{1}}^{\tau_{2}}\left|\mu_{2}(q)\right| u_{t}(x, t-q) d q & (x, t) \in \Omega \times(0, \infty), \\ =b|u|^{p-2} u, & x \in \partial \Omega, \\ u(x, t)=0, & (x, t) \in \Omega \times\left(0, \tau_{2}\right), \\ u_{t}(x,-t)=f_{0}(x, t), & x \in \Omega, \\ u(x, 0)=u_{0}(x), u_{t}(x, 0)=u_{1}(x), & \end{cases}
$$

where $b, \mu_{1}>0, p>2$ and $\tau_{1}, \tau_{2}$ are the time delay with $0 \leq \tau_{1}<\tau_{2}, \mu_{2}$ is an $L^{\infty}$ function, and $g$ is a differentiable function under the assumptions (A1), (A2), and (A3). $M(s)$ is a nonnegative function of $C^{1}$ for $s \geq 0$ satisfy, $M(s)=m_{0}+\alpha s^{\gamma}$, $m_{0}>0, \alpha \geq 0$ and $\gamma \geq 0$, specially we take $M(s)=1+s^{\gamma}$ where $m_{0}=1, \alpha=1$.

Problems about the mathematical behavior of solutions for PDEs with time delay effects have become interesting for many authors mainly because time delays often appear in many practical problems such as thermal, economic phenomena, biological, chemical, physical, electrical engineering systems, mechanical applications and medicine. Moreover, it is well known that delay effects may destroy the stabilizing properties of a well-behaved system. In the literature, there are several examples that illustrate how time delays destabilize some internal or boundary control system $[5,6]$. Viscous materials are the opposite of elastic materials that posses the ability to dissipate and store the mechanical energy. The mechanical

Date: Received: 2021-06-25; Accepted: 2021-07-30.

2000 Mathematics Subject Classification. 35B44; 35L05, 35L70.

Key words and phrases. Distributed delay, Nonexistence, Kirchhoff-type viscoelastic equation. 
properties of these viscous substances are of great importance when they seem in many natural sciences applications [2]. The problem (1.1) is a general form of a model introduced by Kirchhoff [7]. To be more precise, Kirchhoff recommended a model denoted by the equation for $f=g=0$,

$$
\rho h \frac{\partial^{2} u}{\partial t^{2}}+\delta \frac{\partial u}{\partial t}+g\left(\frac{\partial u}{\partial t}\right)=\left\{\rho_{0}+\frac{E h}{2 L} \int_{0}^{L}\left(\frac{\partial u}{\partial x}\right)^{2} d x\right\} \frac{\partial^{2} u}{\partial x^{2}}+f(u)
$$

for $0<x<L, t \geq 0$, where $u(x, t)$ is the lateral displacement, $E$ is the Young modulus, $\rho$ is the mass density, $h$ is the cross-section area, $L$ is the lenght, $\rho_{0}$ is the initial axial tension, $\delta$ is the resistance modulus, and $f$ and $g$ are the external forces. Furthermore, (1.2) is called a degenerate equation when $\rho_{0}=0$ and nondegenerate one when $\rho_{0}>0$.

In 1986, Datko et al. [4] indicated that delay is a source of instability. In [9], Nicaise and Pignotti considered the following wave equation with a linear damping and delay term

$$
u_{t t}-\Delta u+\mu_{1} u_{t}(x, t)+\mu_{2} u_{t}(x, t-\tau)=0 .
$$

They obtained some stability results in the case $0<\mu_{2}<\mu_{1}$. In the absence of delay, Zuazua [23] looked into exponentially stability for the equation (1.3).

$\mathrm{Wu}$ and Tsai [24], considered the following Kirchhoff-type equation

$$
u_{t t}-M\left(\|\nabla u\|_{2}^{2}\right) \Delta u+\left|u_{t}\right|^{r-2} u_{t}=|u|^{p-2} u
$$

with the positive upper bounded initial energy and they obtained the blow-up of solutions for the equation (1.4). In 2013, Ye [22], considered the global existence results by constructing a stable set in $H_{0}^{1}(\Omega)$ and showed the decay by using a lemma of Komornik for the nonlinear Kirchhoff-type equation (1.4) with dissipative term.

When $M(s)=1$, the equation (1.1) becomes the following form

$$
\begin{aligned}
& u_{t t}-\Delta u-\omega \Delta u_{t}+\int_{0}^{t} g(t-s) \Delta u(s) d s \\
& +\mu_{1} u_{t}+\int_{\tau_{1}}^{\tau_{2}}\left|\mu_{2}(\rho)\right| u_{t}(x, t-\rho) d \rho \\
& =b|u|^{p-2} u .
\end{aligned}
$$

In [2], Choucha et al. obtained the blow-up of solutions under appropriate conditions of the equation (1.5). In [3], the authors showed the exponential growth of solution for the equation (1.5). In recent years, some other authors investigate hyperbolic type equations (see $[10,11,12,13,14,15,16,17,18,19,20,21]$ ).

In this paper, we consider the Kirchhoff-type $\left(M\left(\|\nabla u\|^{2}\right)\right)$ viscoelastic equation (1.1) with distributed delay $\left(\int_{\tau_{1}}^{\tau_{2}}\left|\mu_{2}(q)\right| u_{t}(x, t-q) d q\right)$ and source $\left(b|u|^{p-2} u\right)$ terms. Our aim is to obtain the nonexistence of global solutions for the equation (1.1).

The paper is organized as follows: In section 2, we give some materials that will be used later. In section 3 , we state and prove our main result. 


\section{Preliminaries}

In this part, we give materials for the proof of our result. As usual, the notation $\|\cdot\|_{p}$ denotes $L^{p}$ norm, and (., .) is the $L^{2}$ inner product. In particular, we write $\|$. instead of $\|\cdot\|_{2}$.

Now, we denote some assumptions used in this paper:

(A1) $g: R_{+} \rightarrow R_{+}$is a decreasing and differentiable function, that

$$
g(t) \geq 0,1-\int_{0}^{\infty} g(s) d s=l>0 .
$$

(A2) There exists a constant $\xi>0$, that

$$
g^{\prime}(t) \leq-\xi g(t), t \geq 0 .
$$

(A3) $\mu_{2}:\left[\tau_{1}, \tau_{2}\right] \rightarrow R$ is an $L^{\infty}$ function, that

$$
\left(\frac{2 \delta-1}{2}\right) \int_{\tau_{1}}^{\tau_{2}}\left|\mu_{2}(\rho)\right| d \rho \leq \mu_{1}, \delta>\frac{1}{2} .
$$

Let $B_{p}>0$ be the constant satisfies [1]

$$
\|v\|_{p} \leq B_{p}\|\nabla v\|_{p}, \text { for } v \in H_{0}^{1}(\Omega) .
$$

It holds

$$
\begin{aligned}
\int_{0}^{t} g(t-s)\left(\nabla u(s), \nabla u_{t}(t)\right) d s= & -\frac{1}{2} g(t)\|\nabla u(t)\|^{2}+\frac{1}{2}\left(g^{\prime} o \nabla u\right)(t) \\
& -\frac{1}{2} \frac{d}{d t}\left[(g o \nabla u)(t)-\left(\int_{0}^{t} g(s) d s\right)\|\nabla u(t)\|^{2}\right],
\end{aligned}
$$

where

$$
(g \circ \nabla u)(t)=\int_{\Omega} \int_{0}^{t} g(t-s)|\nabla u(t)-\nabla u(s)|^{2} d s .
$$

Firstly, as in [8], we introduce the new variable

$$
y(x, \rho, q, t)=u_{t}(x, t-q \rho),
$$

thus, we get

$$
\left\{\begin{array}{c}
q y_{t}(x, \rho, q, t)+y_{\rho}(x, \rho, q, t)=0 \\
y(x, 0, q, t)=u_{t}(x, t) .
\end{array}\right.
$$

Hence, problem (1.1) is equivalent to:

$$
\left\{\begin{array}{l}
u_{t t}-M\left(\|\nabla u\|^{2}\right) \Delta u+\int_{0}^{t} g(t-s) \Delta u(s) d s \\
+\mu_{1} u_{t}+\int_{\tau_{1}}^{\tau_{2}}\left|\mu_{2}(q)\right||y(x, 1, q, t)| d q \\
=b|u|^{p-2} u, \quad x \in \Omega, t>0 \\
q y_{t}(x, \rho, q, t)+y_{\rho}(x, \rho, q, t)=0
\end{array}\right.
$$

with initial and boundary conditions

$$
\left\{\begin{array}{l}
u(x, t)=0, \quad x \in \partial \Omega, \\
y(x, \rho, q, 0)=f_{0}(x, q \rho), \\
u(x, 0)=u_{0}(x), u_{t}(x, 0)=u_{1}(x)
\end{array}\right.
$$

where

$$
(x, \rho, q, t) \in \Omega \times(0,1) \times\left(\tau_{1}, \tau_{2}\right) \times(0, \infty) .
$$


Theorem 2.1. Suppose that (2.1), (2.2) and (2.3) hold. Let

$$
\left\{\begin{array}{l}
p \geq 2, \quad n=1,2, \\
2<p<\frac{2 n-2}{n-2}, n \geq 3 .
\end{array}\right.
$$

Thus, for any initial data

$$
\left(u_{0}, u_{1}, f_{0}\right) \in H_{0}^{1}(\Omega) \times H_{0}^{1}(\Omega) \times L^{2}\left(\Omega \times(0,1) \times\left(\tau_{1}, \tau_{2}\right)\right),
$$

the problem (2.8)-(2.9) has a unique solution

$$
u \in C\left([0, T] ; H_{0}^{1}(\Omega)\right),
$$

for some $T>0$.

Now, we define the energy functional as follows:

Lemma 2.2. Suppose that (2.1), (2.2), (2.3) and (2.10) hold. Let u be a solution of (2.8). Then, $E(t)$ is nonincreasing, such that

$$
\begin{aligned}
E(t)= & \frac{1}{2}\left\|u_{t}\right\|^{2}+\frac{1}{2}\left(1-\int_{0}^{t} g(s) d s\right)\|\nabla u\|^{2} \\
& +\frac{1}{2(\gamma+1)}\|\nabla u\|^{2(\gamma+1)}+\frac{1}{2}(g o \nabla u)(t) \\
& +\frac{1}{2} \int_{\Omega} \int_{0}^{1} \int_{\tau_{1}}^{\tau_{2}} q\left|\mu_{2}(q)\right|\left|y^{2}(x, \rho, q, t)\right| d q d \rho d x-\frac{b}{p}\|u\|_{p}^{p},
\end{aligned}
$$

which satisfies

$$
E^{\prime}(t) \leq-c_{1}\left(\left\|u_{t}\right\|^{2}+\int_{\Omega} \int_{\tau_{1}}^{\tau_{2}}\left|\mu_{2}(q)\right|\left|y^{2}(x, 1, q, t)\right| d q d x\right) .
$$

Proof. By multiplying the first equation of $(2.8)$ by $u_{t}$ and integrating over $\Omega$, we obtain

$$
\begin{aligned}
\frac{d}{d t}\left\{\frac{1}{2}\left\|u_{t}\right\|^{2}+\frac{1}{2}\left(1-\int_{0}^{t} g(s) d s\right)\|\nabla u\|^{2}\right. \\
\left.+\frac{1}{2(\gamma+1)}\|\nabla u\|^{2(\gamma+1)}+\frac{1}{2}(g o \nabla u)(t)-\frac{b}{p}\|u\|_{p}^{p}\right\} \\
=-\mu_{1}\left\|u_{t}\right\|^{2}-\int_{\Omega} u_{t} \int_{\tau_{1}}^{\tau_{2}}\left|\mu_{2}(q)\right||y(x, 1, q, t)| d q d x \\
+\frac{1}{2}\left(g^{\prime} o \nabla u\right)(t)-\frac{1}{2} g(t)\|\nabla u\|^{2},
\end{aligned}
$$

and

$$
\begin{array}{r}
\frac{d}{d t} \frac{1}{2} \int_{\Omega} \int_{0}^{1} \int_{\tau_{1}}^{\tau_{2}} q\left|\mu_{2}(q)\right|\left|y^{2}(x, \rho, q, t)\right| d q d \rho d x \\
\quad=-\frac{1}{2} \int_{\Omega} \int_{0}^{1} \int_{\tau_{1}}^{\tau_{2}} 2\left|\mu_{2}(q)\right| y y_{\rho} d q d \rho d x \\
\quad=\frac{1}{2} \int_{\Omega} \int_{\tau_{1}}^{\tau_{2}}\left|\mu_{2}(q)\right|\left|y^{2}(x, 0, q, t)\right| d q d x \\
\quad-\frac{1}{2} \int_{\Omega} \int_{\tau_{1}}^{\tau_{2}}\left|\mu_{2}(q)\right|\left|y^{2}(x, 1, q, t)\right| d q d x \\
\quad=\frac{1}{2}\left(\int_{\tau_{1}}^{\tau_{2}}\left|\mu_{2}(q)\right| d q\right)\left\|u_{t}\right\|^{2} \\
\quad-\frac{1}{2} \int_{\Omega} \int_{\tau_{1}}^{\tau_{2}}\left|\mu_{2}(q)\right|\left|y^{2}(x, 1, q, t)\right| d q d x .
\end{array}
$$


Therefore,

$$
\begin{aligned}
\frac{d}{d t} E(t)= & -\mu_{1}\left\|u_{t}\right\|^{2}-\int_{\Omega} \int_{\tau_{1}}^{\tau_{2}}\left|\mu_{2}(q)\right|\left|u_{t} y(x, 1, q, t)\right| d q d x+\frac{1}{2}\left(g^{\prime} o \nabla u\right)(t) \\
& -\frac{1}{2} g(t)\|\nabla u\|^{2}+\frac{1}{2}\left(\int_{\tau_{1}}^{\tau_{2}}\left|\mu_{2}(q)\right| d q\right)\left\|u_{t}\right\|^{2} \\
& -\frac{1}{2} \int_{\Omega} \int_{\tau_{1}}^{\tau_{2}}\left|\mu_{2}(q)\right|\left|y^{2}(x, 1, q, t)\right| d q d x .
\end{aligned}
$$

By using (2.13) and (2.14), we obtain (2.11). Utilizing Young's inequality, (2.1), (2.2), (2.3) and (2.15), we get (2.12). Hence, we complete the proof.

Lemma 2.3. [2] There exists $c>0$, depending on $\Omega$ only, such that

$$
\left(\int_{\Omega}|u|^{p} d x\right)^{s / p} \leq c\left[\|\nabla u\|^{2}+\|u\|_{p}^{p}\right]
$$

for all $u \in L^{p+1}(\Omega)$ and $2 \leq s \leq p$.

Using the fact that $\|u\|_{2}^{2} \leq c\|u\|_{p}^{2} \leq c\left(\|u\|_{p}^{p}\right)^{2 / p}$, we have the corollary as follows:

Corollary 2.3.1. There exists $C>0$, depending on $\Omega$ only, that

$$
\|u\|_{2}^{2} \leq c\left[\|\nabla u\|_{2}^{4 / p}+\left(\|u\|_{p}^{p}\right)^{2 / p}\right] .
$$

Lemma 2.4. [2] There exists $C>0$, depending on $\Omega$ only, such that

$$
\|u\|_{p}^{s} \leq C\left[\|\nabla u\|^{2}+\|u\|_{p}^{p}\right],
$$

for all $u \in L^{p+1}(\Omega)$ and $2 \leq s \leq p$.

Now, we define the functional as follows:

$$
\begin{aligned}
H(t)= & -E(t) \\
= & \frac{b}{p}\|u\|_{p}^{p}-\frac{1}{2}\left\|u_{t}\right\|^{2} \\
& -\frac{1}{2}\left(1-\int_{0}^{t} g(s) d s\right)\|\nabla u\|^{2} \\
& -\frac{1}{2(\gamma+1)}\|\nabla u\|^{2(\gamma+1)}-\frac{1}{2}(g o \nabla u)(t) \\
& -\frac{1}{2} \int_{\Omega} \int_{0}^{1} \int_{\tau_{1}}^{\tau_{2}} q\left|\mu_{2}(q)\right|\left|y^{2}(x, \rho, q, t)\right| d q d \rho d x .
\end{aligned}
$$

\section{Nonexistence of SOlutions}

In this part, we obtain the nonexistence of global solutions for the problem (2.8)-(2.9).

Theorem 3.1. Suppose that (2.1)-(2.3) and (2.10) hold. Suppose further that $E(0)<0$ holds. Then, the solution of the problem (2.8)-(2.9) blows up in finite time. 
Proof. By (2.11), we get

$$
E(t) \leq E(0) \leq 0
$$

Hence

$$
\begin{aligned}
H^{\prime}(t) & =-E^{\prime}(t) \\
& \geq c_{1}\left(\left\|u_{t}\right\|^{2}+\int_{\Omega} \int_{\tau_{1}}^{\tau_{2}}\left|\mu_{2}(q)\right|\left|y^{2}(x, 1, q, t)\right| d q d x\right) \\
& \geq c_{1} \int_{\Omega} \int_{\tau_{1}}^{\tau_{2}}\left|\mu_{2}(q)\right|\left|y^{2}(x, 1, q, t)\right| d q d x \geq 0,
\end{aligned}
$$

and

$$
0 \leq H(0) \leq H(t) \leq \frac{b}{p}\|u\|_{p}^{p}
$$

Set

$$
\mathcal{K}(t)=H^{1-\alpha}(t)+\varepsilon \int_{\Omega} u u_{t} d x+\frac{\varepsilon \mu_{1}}{2} \int_{\Omega} u^{2} d x,
$$

here $\varepsilon>0$ to be specified later and

$$
\frac{2(p-2)}{p^{2}}<\alpha<\frac{p-2}{2 p}<1
$$

We multiply the first equation of (2.8) by $u$ and with a derivative of (3.4), to obtain

$$
\begin{aligned}
\mathcal{K}^{\prime}(t)= & (1-\alpha) H^{-\alpha}(t) H^{\prime}(t) \\
& +\varepsilon\left\|u_{t}\right\|^{2}+\varepsilon \int_{\Omega} \nabla u \int_{0}^{t} g(t-s) \nabla u(s) d s d x \\
& -\varepsilon\|\nabla u\|^{2}-\varepsilon\|\nabla u\|^{2(\gamma+1)}+\varepsilon b \int_{\Omega}|u|^{p} d x \\
& -\varepsilon \int_{\Omega} \int_{\tau_{1}}^{\tau_{2}}\left|\mu_{2}(q)\right||u y(x, 1, q, t)| d q d x .
\end{aligned}
$$

By using

$$
\begin{aligned}
& \varepsilon \int_{\Omega} \int_{\tau_{1}}^{\tau_{2}}\left|\mu_{2}(q)\right||u y(x, 1, q, t)| d q d x \\
\leq & \varepsilon\left\{\delta_{1}\left(\int_{\tau_{1}}^{\tau_{2}}\left|\mu_{2}(q)\right| d q\right)\|u\|^{2}\right. \\
& \left.+\frac{1}{4 \delta_{1}} \int_{\Omega} \int_{\tau_{1}}^{\tau_{2}}\left|\mu_{2}(q)\right|\left|y^{2}(x, 1, q, t)\right| d q d x\right\},
\end{aligned}
$$


NONEXISTENCE OF GLOBAL SOLUTIONS FOR A KIRCHHOFF-TYPE VISCOELASTIC EQUATIØN

and

$$
\begin{aligned}
& \varepsilon \int_{0}^{t} g(t-s) d s \int_{\Omega} \nabla u \nabla u(s) d x d s \\
= & \varepsilon \int_{0}^{t} g(t-s) d s \int_{\Omega} \nabla u(\nabla u(s)-\nabla u(t)) d x d s \\
& +\varepsilon \int_{0}^{t} g(s) d s\|\nabla u\|^{2} \\
\geq & \frac{\varepsilon}{2} \int_{0}^{t} g(s) d s\|\nabla u\|^{2}-\frac{\varepsilon}{2}(g o \nabla u)(t) .
\end{aligned}
$$

By (3.6), we get

$$
\begin{aligned}
\mathcal{K}^{\prime}(t) \geq & (1-\alpha) H^{-\alpha}(t) H^{\prime}(t)+\varepsilon\left\|u_{t}\right\|^{2}-\varepsilon\left(1-\frac{1}{2} \int_{0}^{t} g(s) d s\right)\|\nabla u\|^{2} \\
& -\varepsilon\|\nabla u\|^{2(\gamma+1)}+\varepsilon b\|u\|_{p}^{p}-\varepsilon \delta_{1}\left(\int_{\tau_{1}}^{\tau_{2}}\left|\mu_{2}(q)\right| d q\right)\|u\|^{2} \\
& -\frac{\varepsilon}{4 \delta_{1}} \int_{\Omega} \int_{\tau_{1}}^{\tau_{2}}\left|\mu_{2}(q)\right|\left|y^{2}(x, 1, q, t)\right| d q d x+\frac{\varepsilon}{2}(g o \nabla u)(t) .
\end{aligned}
$$

By using (3.2) and setting $\delta_{1}$ such that, $\frac{1}{4 \delta_{1} c_{1}}=\kappa H^{-\alpha}(t)$, we obtain

$$
\begin{aligned}
\mathcal{K}^{\prime}(t) \geq & {[(1-\alpha)-\varepsilon \kappa] H^{-\alpha}(t) H^{\prime}(t)+\varepsilon\left\|u_{t}\right\|^{2} } \\
& -\varepsilon\left[\left(1-\frac{1}{2} \int_{0}^{t} g(s) d s\right)\right]\|\nabla u\|^{2}-\varepsilon\|\nabla u\|^{2(\gamma+1)} \\
+ & +\varepsilon b\|u\|_{p}^{p}-\varepsilon \frac{H^{\alpha}(t)}{4 c_{1} \kappa}\left(\int_{\tau_{1}}^{\tau_{2}}\left|\mu_{2}(q)\right| d q\right)\|u\|^{2}+\frac{\varepsilon}{2}(g o \nabla u)(t) .
\end{aligned}
$$

For $0<a<1$, by $(2.19)$

$$
\begin{aligned}
\varepsilon b\|u\|_{p}^{p}= & \varepsilon p(1-a) H(t)+\frac{\varepsilon p(1-a)}{2}\left\|u_{t}\right\|^{2}+\varepsilon b a\|u\|_{p}^{p} \\
& +\frac{\varepsilon p(1-a)}{2}\left(1-\int_{0}^{t} g(s) d s\right)\|\nabla u\|^{2} \\
& +\frac{\varepsilon p(1-a)}{2(\gamma+1)}\|\nabla u\|^{2(\gamma+1)}+\frac{\varepsilon}{2} p(1-a)(g o \nabla u)(t) \\
& +\frac{\varepsilon p(1-a)}{2} \int_{\Omega} \int_{0}^{1} \int_{\tau_{1}}^{\tau_{2}} q\left|\mu_{2}(q)\right|\left|y^{2}(x, \rho, q, t)\right| d q d \rho d x
\end{aligned}
$$


with (3.10), it gives

$$
\begin{aligned}
\mathcal{K}^{\prime}(t) \geq & {[(1-\alpha)-\varepsilon \kappa] H^{-\alpha}(t) H^{\prime}(t)+\varepsilon\left[\frac{p(1-a)}{2}+1\right]\left\|u_{t}\right\|^{2} } \\
& +\varepsilon\left[\left(\frac{p(1-a)}{2}\right)\left(1-\int_{0}^{t} g(s) d s\right)-\left(1-\frac{1}{2} \int_{0}^{t} g(s) d s\right)\right]\|\nabla u\|^{2} \\
& +\varepsilon\left(\frac{p(1-a)}{2(\gamma+1)}-1\right)\|\nabla u\|^{2(\gamma+1)}-\varepsilon \frac{H^{\alpha}(t)}{4 c_{1} \kappa}\left(\int_{\tau_{1}}^{\tau_{2}}\left|\mu_{2}(q)\right| d q\right)\|u\|^{2} \\
& +\frac{\varepsilon p(1-a)}{2} \int_{\Omega} \int_{0}^{1} \int_{\tau_{1}}^{\tau_{2}} q\left|\mu_{2}(q)\right|\left|y^{2}(x, \rho, q, t)\right| d q d \rho d x \\
& +\varepsilon p(1-a) H(t)+\varepsilon b a\|u\|_{p}^{p}+\frac{\varepsilon}{2}(p(1-a)+1)(g o \nabla u)(t) .
\end{aligned}
$$

By using (2.17), (3.3) and Young's inequality, we obtain

$$
\begin{aligned}
H^{\alpha}(t)\|u\|_{2}^{2} & \leq\left(b \int_{\Omega}|u|^{p} d x\right)^{\alpha}\|u\|_{2}^{2} \\
& \leq c\left\{\left(\int_{\Omega}|u|^{p} d x\right)^{\alpha+2 / p}+\left(\int_{\Omega}|u|^{p} d x\right)^{\alpha}\|\nabla u\|_{2}^{4 / p}\right\} \\
& \leq c\left\{\left(\int_{\Omega}|u|^{p} d x\right)^{(p \alpha+2) / p}+\|\nabla u\|_{2}^{2}+\left(\int_{\Omega}|u|^{p} d x\right)^{p \alpha /(p-2)}\right\} .
\end{aligned}
$$

By exploiting (3.5), we obtain

$$
2<\alpha p+2 \leq p \text { and } 2<\frac{\alpha p^{2}}{p-2} \leq p .
$$

As a result, by Lemma 2.2, such that

$$
H^{\alpha}(t)\|u\|_{2}^{2} \leq c\left(\|u\|_{p}^{p}+\|\nabla u\|_{2}^{2}\right) .
$$

By combining (3.12) and (3.14), we have

$$
\begin{aligned}
\mathcal{K}^{\prime}(t) \geq & {[(1-\alpha)-\varepsilon \kappa] H^{-\alpha}(t) H^{\prime}(t) } \\
& +\varepsilon\left[\frac{p(1-a)}{2}+1\right]\left\|u_{t}\right\|^{2}+\frac{\varepsilon}{2}(p(1-a)+1)(g o \nabla u)(t) \\
& +\varepsilon\left\{\left(\frac{p(1-a)}{2}-1\right)-\int_{0}^{t} g(s) d s\left(\frac{p(1-a)-1}{2}\right)\right. \\
& \left.-\frac{c}{4 c_{1} \kappa}\left(\int_{\tau_{1}}^{\tau_{2}}\left|\mu_{2}(q)\right| d q\right)\right\}\|\nabla u\|^{2} \\
& +\varepsilon\left[a b-\frac{c}{4 c_{1} \kappa}\left(\int_{\tau_{1}}^{\tau_{2}}\left|\mu_{2}(q)\right| d q\right)\right]\|u\|_{p}^{p} \\
& +\varepsilon\left(\frac{p(1-a)}{2(\gamma+1)}-1\right)\|\nabla u\|^{2(\gamma+1)}+\varepsilon p(1-a) H(t) \\
& +\frac{\varepsilon p(1-a)}{2} \int_{\Omega} \int_{0}^{1} \int_{\tau_{1}}^{\tau_{2}} q\left|\mu_{2}(q)\right|\left|y^{2}(x, \rho, q, t)\right| d q d \rho d x .
\end{aligned}
$$


NONEXISTENCE OF GLOBAL SOLUTIONS FOR A KIRCHHOFF-TYPE VISCOELASTIC EQUATIQnף

Taking $a>0$ small enough, that

$$
\alpha_{1}=\frac{p(1-a)}{2}-1>0
$$

and suppose

$$
\int_{0}^{\infty} g(s) d s<\frac{\frac{p(1-a)}{2}-1}{\left(\frac{p(1-a)}{2}-\frac{1}{2}\right)}=\frac{2 \alpha_{1}}{2 \alpha_{1}+1} .
$$

Choosing $\kappa$ such that,

$$
\begin{aligned}
\alpha_{2}= & \left(\frac{p(1-a)}{2}-1\right)-\int_{0}^{t} g(s) d s\left(\frac{p(1-a)-1}{2}\right) \\
& -\frac{c}{4 c_{1} \kappa}\left(\int_{\tau_{1}}^{\tau_{2}}\left|\mu_{2}(q)\right| d q\right) \\
> & 0
\end{aligned}
$$

and

$$
\alpha_{3}=a b-\frac{c}{4 c_{1} \kappa}\left(\int_{\tau_{1}}^{\tau_{2}}\left|\mu_{2}(q)\right| d q\right)>0 \text { and } \frac{p(1-a)}{2(\gamma+1)}-1>0 .
$$

Fixing $\kappa$ and $a$, we have $\varepsilon$ small enough,

$$
\alpha_{4}=(1-\alpha)-\varepsilon \kappa>0 .
$$

Hence, for some $\beta>0$, (3.15) becomes

$$
\begin{aligned}
\mathcal{K}^{\prime}(t) \geq & \beta\left\{H(t)+\left\|u_{t}\right\|^{2}+\|\nabla u\|^{2}+\|\nabla u\|^{2(\gamma+1)}+(g o \nabla u)(t)\right. \\
& \left.+\|u\|_{p}^{p}+\int_{\Omega} \int_{0}^{1} \int_{\tau_{1}}^{\tau_{2}} q\left|\mu_{2}(q)\right|\left|y^{2}(x, \rho, q, t)\right| d q d \rho d x\right\} .
\end{aligned}
$$

Therefore,

$$
\mathcal{K}(t) \geq \mathcal{K}(0)>0, t>0
$$

Now, utilizing Holder's and Young's inequalities, we obtain

$$
\begin{aligned}
\|u\|_{2} & =\left(\int_{\Omega} u^{2} d x\right)^{\frac{1}{2}} \\
& \leq\left[\left(\int_{\Omega}\left(|u|^{2}\right)^{p / 2} d x\right)^{\frac{2}{p}}\left(\int_{\Omega} 1 d x\right)^{1-\frac{2}{p}}\right]^{\frac{1}{2}} \\
& \leq C\|u\|_{p}
\end{aligned}
$$

and

$$
\left|\int_{\Omega} u u_{t} d x\right| \leq\left\|u_{t}\right\|_{2}\|u\|_{2} \leq c\left\|u_{t}\right\|_{2}\|u\|_{p}
$$

Hence,

$$
\begin{aligned}
\left|\int_{\Omega} u u_{t} d x\right|^{\frac{1}{1-\alpha}} & \leq c\left\|u_{t}\right\|_{2}^{\frac{1}{1-\alpha}}\|u\|_{p}^{\frac{1}{1-\alpha}} \\
& \leq c\left[\left\|u_{t}\right\|_{2}^{\frac{\theta}{1-\alpha}}+\|u\|_{p}^{\frac{\mu}{1-\alpha}}\right],
\end{aligned}
$$


here $\frac{1}{\mu}+\frac{1}{\theta}=1$. Taking $\theta=2(1-\alpha)$, we have

$$
\frac{\mu}{1-\alpha}=\frac{2}{1-2 \alpha} \leq p \text {. }
$$

For $s=\frac{2}{(1-2 \alpha)}$, we get

$$
\left|\int_{\Omega} u u_{t} d x\right|^{\frac{1}{1-\alpha}} \leq c\left(\left\|u_{t}\right\|_{2}^{2}+\|u\|_{p}^{s}\right) .
$$

Hence, Lemma 2.3 gives

$$
\begin{aligned}
\left|\int_{\Omega} u u_{t} d x\right|^{\frac{1}{1-\alpha}} & \leq c\left[\left\|u_{t}\right\|_{2}^{2}+\|u\|_{p}^{p}+\|\nabla u\|_{2}^{2}\right] \\
& \leq c\left[\left\|u_{t}\right\|_{2}^{2}+\|u\|_{p}^{p}+\|\nabla u\|_{2}^{2}+\|\nabla u\|^{2(\gamma+1)}+(g o \nabla u)(t)\right] .
\end{aligned}
$$

Therefore,

$$
\begin{aligned}
\mathcal{K}^{\frac{1}{1-\alpha}}(t) & =\left(H(t)^{1-\alpha}+\varepsilon \int_{\Omega} u u_{t} d x+\frac{\varepsilon \mu_{1}}{2} \int_{\Omega} u^{2} d x\right)^{\frac{1}{1-\alpha}} \\
& \leq c\left[H(t)+\left|\int_{\Omega} u u_{t} d x\right|^{\frac{1}{1-\alpha}}+\|u\|_{2}^{\frac{2}{1-\alpha}}+\|\nabla u\|_{2}^{\frac{2}{1-\alpha}}\right] \\
& \leq c\left[H(t)+\left\|u_{t}\right\|^{2}+\|u\|_{p}^{p}+\|\nabla u\|^{2}+\|\nabla u\|^{2(\gamma+1)}+(g o \nabla u)(t)\right] .
\end{aligned}
$$

By (3.17) and (3.22), we obtain

$$
\mathcal{K}^{\prime}(t) \geq \lambda \mathcal{K}^{\frac{1}{1-\alpha}}(t)
$$

here $\lambda>0$, which depends on $\beta$ and $c$. An integration of (3.23), we get

$$
\mathcal{K}^{\frac{\alpha}{1-\alpha}}(t) \geq \frac{1}{\mathcal{K}^{\frac{-\alpha}{1-\alpha}}(0)-\lambda \frac{\alpha}{(1-\alpha)} t} .
$$

Therefore, $\mathcal{K}(t)$ blows up in time

$$
T \leq T^{*}=\frac{1-\alpha}{\lambda \alpha \mathcal{K}^{\alpha /(1-\alpha)}(0)}
$$

Then, the proof is completed.

\section{Conclusion}

In recent years, there has been published much work concerning the wave equations (Kirchhoff, Petrovsky, Bessel,... etc.) with different state of delay time (constant delay, time-varying delay,... etc.). However, to the best of our knowledge, there were no nonexistence of global results for the Kirchhoff-type viscoleastic equation with distributed delay and source terms. We have been obtained the nonexistence of global solutions under suitable conditions. 


\section{ACKNOWLEDGMents}

The authors would like to thank the reviewers and editors of Journal of Universal Mathematics.

\section{Funding}

The authors are grateful to DUBAP (ZGEF.20.009) for research funds.

\section{The Declaration of Conflict of Interest/ Common Interest}

The author(s) declared that no conflict of interest or common interest

\section{The Declaration of Ethics Committee Approval}

This study does not be necessary ethical committee permission or any special permission.

\section{The Declaration of Research and Publication Ethics}

The author(s) declared that they comply with the scientific, ethical, and citation rules of Journal of Universal Mathematics in all processes of the study and that they do not make any falsification on the data collected. Besides, the author(s) declared that Journal of Universal Mathematics and its editorial board have no responsibility for any ethical violations that may be encountered and this study has not been evaluated in any academic publication environment other than Journal of Universal Mathematics.

\section{REFERENCES}

[1] R.A. Adams, J.J.F. Fournier, Sobolev Spaces, Academic Press, (2003).

[2] A. Choucha, D. Ouchenane and S. Boulaaras, Blow-up of a nonlinear viscoelastic wave equation with distributed delay combined with strong damping and source terms, J. Nonlinear Funct. Anal., 2020, pp. 1-10 (2020).

[3] A. Choucha, D. Ouchenane and K. Zennir, Exponential growth of solution with $L_{p}$-norm for class of non-linear viscoelastic wave equation with distributed delay term for large initial data, Open J. Math. Anal., 3(1), pp. 76-83 (2020).

[4] R. Datko, J. Lagnese and M.P. Polis, An example on the effect of time delays in boundary feedback stabilization of wave equations, SICON, 24(1), pp. 152-156 (1986).

[5] J.K. Hale, S.M. Verduyn Lunel, Introduction to Functional-Differential Equations, Appl. Math. Sci., 99, x+447, (Springer-Verlag, New York), (1993).

[6] M. Kafini, S.A. Messaoudi, A blow-up result in a nonlinear wave equation with delay, Mediterr. J. Math., 13, pp. 237-247 (2016).

[7] G. Kirchhoff, Vorlesungen über Mechanik, 3rd. ed., Teubner, Leipzig, (1883).

[8] S. Nicaise, C. Pignotti, Stabilization of the wave equation with boundary or internal distributed delay, Differ. Integral Equ., 21, pp. 935-958 (2008).

[9] S. Nicaise, C. Pignotti, Stability and instability results of the wave equation with a delay term in the boundary or internal feedbacks, SIAM J. Control Optim, 45(5), pp. 1561-1585 (2006).

[10] E. Pişkin, H. Yüksekkaya, Local existence and blow up of solutions for a logarithmic nonlinear viscoelastic wave equation with delay, Comput. Methods Differ. Equ., 9(2), pp. 623-636 (2021).

[11] E. Pişkin, H. Yüksekkaya, Nonexistence of global solutions of a delayed wave equation with variable-exponents, Miskolc Math. Notes, pp. 1-19. (Accepted)

[12] E. Pişkin, H. Yüksekkaya, Blow up of solution for a viscoelastic wave equation with mLaplacian and delay terms, Tbil. Math. J., SI (7),pp. 21-32 (2021). 
[13] E. Pişkin, H. Yüksekkaya, Blow-up of solutions for a logarithmic quasilinear hyperbolic equation with delay term, J. Math. Anal., 12(1), pp. 56-64 (2021).

[14] E. Pişkin, H. Yüksekkaya, Non-existence of solutions for a Timoshenko equations with weak dissipation, Math. Morav., 22 (2), pp. 1-9 (2018).

[15] E. Pişkin and H. Yüksekkaya, Decay of solutions for a nonlinear Petrovsky equation with delay term and variable exponents, The Aligarh Bull. of Maths., 39(2),pp. 63-78, (2020).

[16] H. Yüksekkaya, E. Pişkin, Blow up of Solutions for Petrovsky Equation with Delay Term, Journal of Nepal Mathematical Society.4 (1), pp. 76-84 (2021).

[17] E. Pişkin, H. Yüksekkaya, Mathematical behavior of the solutions of a class of hyperbolic-type equation, J. BAUN Inst. Sci. Technol. 20(3), pp. 117-128 (2018).

[18] H. Yüksekkaya, E. Pişkin, S.M. Boulaaras, B.B. Cherif and S.A. Zubair, Existence, Nonexistence, and Stability of Solutions for a Delayed Plate Equation with the Logarithmic Source, Adv. Math. Phys., 2021, pp. 1-11 (2021), https://doi.org/10.1155/2021/8561626.

[19] E. Pişkin, H. Yüksekkaya and N. Mezouar, Growth of Solutions for a Coupled Viscoelastic Kirchhoff System with Distributed Delay Terms, Menemui Matematik (Discovering Mathematics) 43(1) pp. 26-38,(2021).

[20] E. Pişkin, H. Yüksekkaya, Global Attractors for the Higher-Order Evolution Equation, AMNS, 5(1), pp. 195-210 (2020).

[21] S.T. Wu and L.Y. Tsai, Blow-up of solutions for some non-linear wave equations of Kirchhoff type with some dissipation, Nonlinear Anal., 65(2), pp. 243-264 (2006).

[22] Y. Ye, Global existence of solutions and energy decay for a Kirchhoff-type equation with nonlinear dissipation, J. Inequal. Appl., 2013:195(2013).

[23] E. Zuazua, Exponential decay for the semi-linear wave equation with locally disributed damping, Commun. Part. Diff. Eq., 15, pp. 205-235 (1990).

[24] S.T. Wu and L.Y. Tsai, On global existence and blow-up of solutions for an integro-differential equation with strong damping, Taiwanese Journal of Mathematics, 10(4), pp. 979-1014(2006).

(Hazal Yüksekkaya (Corresponding Author)) Dicle University, Department of Mathematics, Diyarbakir, Turkey

Email address: hazally.kaya@gmail.com

(Erhan Pişkin) Dicle University, Department of Mathematics, Diyarbakir, Turkey

Email address: episkin@dicle.edu.tr 